\title{
ACCURACY OF TRANSCUTANEUS BILIRUBIN VALUES: EVALUATION OF THE BEST SITE
}

\author{
P.J. Nader ${ }^{1}$, S.S. Nader ${ }^{1}$, J.G. da Rocha Filho ${ }^{2}$
}

${ }^{l}$ Neonatology, Universidade Luterana do Brasil, Porto Alegre, ${ }^{2}$ Universidade Luterana do Brasil, Canoas, Brazil

Background: Newborns should be monitored to identify who are more likely to develop hyperbilirubinemia. Several methods aimed at assessing bilirubin values. This paper investigates the accuracy, specificity and sensitivity of transcutaneous bilirubin levels in relation to serum bilirubin and evaluates the values from the two sites of measuring transcutaneous bilirubin and notes the behavior of bilirubin according the hours of life.

Method: Prospective study in Rooming-in using transcutaneous measurements of bilirubin and plasma of 209 newborns with suspected jaundice, calculated concordance, sensitivity and specificity of the transcutaneous method.

Results: Assessment of correlation between serum bilirubin and transcutaneous measurement of forehead showing disagreement in only $5.7 \%$, and the dosages of the chest $6.7 \%$. When evaluated the concordance of the two regions to one another disagreement was $4.8 \%$. The ROC curve showed at a dosage transcutaneous $9 \mathrm{mg} / \mathrm{dL}$, sensitivity and specificity of $88.8 \%$ from $31.4 \%$ in the forehead ( $95 \%$ CI 0.65 to 0.79 ) and $83.2 \%$ sensitivity and specificity $36.3 \%$ in the chest $(95 \%$ CI: 0.64 to 0.78$)$. Assessing the behavior of bilirubin in accordance with the hours of life were no significant differences in bilirubin measurements in each region, the forehead was the best dose group $49 \mathrm{~h}-72 \mathrm{~h}$, the chest was already in the groups of $49 \mathrm{~h}-72 \mathrm{~h}$ and $>72 \mathrm{~h}$.

Conclusions: The values obtained by Jaundice Meter JM - 103 are reliable serum values, especially to the value of $9 \mathrm{mg} / \mathrm{dL}$, values above this level should serve as an indication of screening.The forehead was the most confident. 